\title{
¿Administraciones públicas inclusivas?: la implementación de la normativa sobre el acceso de las mujeres con discapacidad al empleo público ${ }^{1}$
}

\section{Inclusive public administrations?: the implementation of the legislation on the access of women with disabilities to public employment}

Rocío Navarro González

Universidad Pablo de Olavide (España)

ORCID: https://orcid.org/0000-0003-1157-3536

rnavgon@upo.es

\begin{abstract}
NOTA BIOGRÁFICA
Profesora ayudante Doctora (acreditada Profesora Contratada Doctora) e investigadora en la Universidad Pablo de Olavide (Sevilla, España). Líneas de investigación: Parte General de la disciplina jurídica del Derecho Administrativo contemporáneo -el acto administrativo-, las potestades administrativas y políticas y servicios sociales.
\end{abstract}

Juana María Ruiloba Núñez

Universidad Pablo de Olavide (España)

ORCID: https://orcid.org/0000-0002-8164-7695

jruiloba@upo.es

\section{NOTA BIOGRÁFICA}

Profesora e investigadora en la Universidad Pablo de Olavide (Sevilla, España). Doctora en Ciencia Política y de la Administración por la Universidad Autónoma de Madrid (2009, UAM) y miembro del Instituto Universitario de Estudios de la Mujer (UAM). Líneas de investigación preferentes: liderazgo político, administración y políticas públicas, estudios de género y gobernanza local.

\section{RESUMEN}

Las Administraciones Públicas tienen un papel relevante en mejorar las oportunidades en el empleo público de las personas con discapacidad. El objetivo central del trabajo es constatar la importancia de apostar efectivamente por administraciones públicas más diversas, inclusivas e igualitarias. La investigación hace un recorrido del grado de implementación de la normativa existente sobre el acceso al empleo público; en concreto del proceso de aplicación de las medidas correctoras de posibles desigualdades que fortalezcan la protección y garanticen la igualdad efectiva de oportunidades en el acceso al empleo del sector público de las mujeres con discapacidad. Pese a la ingente producción normativa, se desprende de este estudio

1 Las autoras agradecen a los dos evaluadores anónimos y editores de la revista la labor de revisión realizada y los comentarios y sugerencias de mejora que han aportado, enriqueciendo la versión definitiva del texto. 
la falta de concreción práctica y la necesidad de una implicación mayor en la implementación y evaluación de las acciones diseñadas. El año 2020 es punto de inflexión para la incorporación de un enfoque más interseccional, concretado en un cambio de paradigma organizacional hacia la diversidad, aún por plasmar.

\title{
PALABRAS CLAVE
}

Empleo público; Administración Pública; Discapacidad; Género; políticas de igualdad de oportunidades.

\begin{abstract}
Public Administrations have a significant role to play in improving opportunities in public employment for people with disabilities. The main objective of this paper is to verify the importance of effectively achieving more diverse, inclusive, and egalitarian public administrations. The research covers the degree of implementation of the existing regulations on access to public employment; specifically, the process of applying corrective measures for possible inequalities that strengthen protection and guarantee effective equality of opportunities in access to public sector employment for women with disabilities. Despite the enormous normative production, this study shows the lack of practical specificity and the need for greater involvement in the implementation and evaluation of the actions design. The year 2020 is a turning point for the incorporation of a more cross-sectional approach, made concrete through a shift in the organisational paradigm towards diversity, which is yet to be realised.
\end{abstract}

\section{KEYWORDS}

Public employment; Public Administration; disability; gender; equal opportunities policy.

\section{SUMARIO}

INTRODUCCIÓN. 1. ADMINISTRACIONES PÚBLICAS INCLUSIVAS: ALGUNOS DEBATES TEÓRICOS. 1.1. ADMINISTRACIONES PÚBLICAS RESPONSABLES Y DIVERSAS. 2. EL MARCO GENERAL DE PROTECCIÓN DE LAS PERSONAS CON DISCAPACIDAD. 3. LA ACCIÓN PÚBLICA DISEÑADA PARA EL ACCESO DE LAS PERSONAS CON DISCAPACIDAD AL EMPLEO PÚBLICO. 3.1. MEDIDAS DE ACCIÓN POSITIVA PARA PROMOVER EL ACCESO AL EMPLEO PÚBLICO. 3.2 OTRAS MEDIDAS QUE FAVORECEN LA INTEGRACIÓN. 4. EL PAPEL DE LA ADMINISTRACIÓN EMPLEADORA Y PROMOTORA DE LA IGUALDAD DE GÉNERO. 4.1. EL RETO DE INCORPORAR SOLUCIONES A LA DISCRIMINACIÓN MÚLTIPLE EN LA NORMATIVA GENERAL DE LA FUNCIÓN PÚBLICA. 4.2. LOS PLANES DE IGUALDAD EN LAS ADMINISTRACIONES PÚBLICAS. 5. RECOMENDACIONES PARA MEJORES PRÁCTICAS EN LAS AA.PP CONCLUSIONES. REFERENCIAS BIBLIOGRÁFICAS.

\section{INTRODUCCIÓN}

El presente trabajo tiene como objetivo central el estudio de las acciones públicas para la promoción y gestión de la diversidad en el interior de las Administraciones públicas (AA.PP). A partir de una descripción sistemática se realiza un recorrido por las políticas de acceso al empleo público y su relación con la igualdad de oportunidades y los efectos sobre el colectivo de mujeres con discapacidad (MCD) en la legislación española a través de una revisión bibliográfica, documental y de la normativa (Grant y Booth, 2009 y Onwuegbuzie y Frels, 2016).

El estudio transciende de los trabajos sobre diversidad en las AA.PP focalizados en el estudio de una sola dimensión de desigualdad (Rice, 2005, p. 88), centrándose en el hecho de cómo dos elementos destacados de la identidad, discapacidad y género, pueden funcionar simultáneamente, en una suerte de discriminación interseccional ${ }^{2}$ y cómo el sector público ha delineado medidas para contrarrestarlo.

2 Las personas pueden «pertenecer a varios grupos en desventaja al mismo tiempo, sufriendo formas agravadas y específicas de discriminación», esto es, discriminación «multiple» o «intersectional» (Rey Martínez, 2008, p. 255). En el lenguaje político y jurídico, la Unión Europea (UE) ha estado usando desde 2007 el concepto «discriminación múltiple», no obstante, las distintas variantes de la discriminación, acumulativa, múltiple, compuesta, se definen, como intersectional discrimination (Comisión Europea, 2007; Makkonen, 2002 , p. 12). De esta manera, en los ambientes académicos es predominante el término interseccional, mientras que en el ámbito de los derechos humanos lo es indistintamente junto con discriminación múltiple. 
La evolución del concepto de discapacidad deambula desde el paradigma tradicional, médico o social, hasta las aportaciones de los enfoques integradores -que inspirarían la Clasificación Internacional del Funcionamiento, de la Discapacidad y de la Salud (CIF), aprobada en 2001 por la Organización Mundial de la Salud (OMS) - y el emergente enfoque de la diversidad funcional. Llewellyn y Hogan (2000) nos advierten del problema de los enfoques individualmente entendidos, puntualizando que «las sociedades avanzadas caminan hacia una generalización de la perspectiva social (de derechos) en todos los órdenes de la vida» ${ }^{3}$.

Frente al entendimiento de la discapacidad como «deficiencia individual» de los primeros tiempos, «el modelo social entiende la discapacidad como el resultado del fracaso de la sociedad para adaptarse a las necesidades de las personas discapacitadas» (Abberley, 1998, p. 78). En este trabajo nos planteamos las limitaciones de adaptación efectiva de las organizaciones públicas.

Dejando a un lado las múltiples definiciones y enfoques de discapacidad (Hahn, 1985; Hahn 1993), la referencia a las personas con discapacidad (PCD) atiende a lo previsto en el Real Decreto Legislativo 1/2013, de 29 de noviembre, por el que se aprueba el Texto Refundido de la Ley General de derechos de las PCD y de su inclusión social (TRLGDPD), la cual dispone que las PCD son «aquellas que presentan deficiencias físicas, mentales, intelectuales o sensoriales, previsiblemente permanentes que, al interactuar con diversas barreras, puedan impedir su participación plena y efectiva en la sociedad, en igualdad de condiciones con los demás. Además de lo establecido en el apartado anterior, y a todos los efectos, tendrán la consideración de PCD aquellas a quienes se les haya reconocido un grado de discapacidad igual o superior al 33 por ciento».

Por otra parte, partiendo de la hipótesis de que el empleo público sirve como «una escalera de oportunidades y un símbolo de la movilidad profesional» (Selden, 2006, p. 917), el hecho que en la administración haya servidores públicos que reflejen las características y los valores específicos de aquellos a quienes sirven, tiene grandes implicaciones más allá de las importantes de carácter individual o de la presencia y la participación de esos grupos potencialmente subrepresentados. Se trata de los efectos sobre las propias estructuras públicas y sobre los impactos en sus resultados a la postre, más justos, más legítimos y efectivos (Conner, 2016, p. 298).

A lo largo de la exposición de este estudio se abordará en el primer capítulo, a partir de la teoría de la burocracia representativa, la responsabilidad pública institucional y el enfoque interseccional, el porqué de la necesaria consideración de estas dimensiones en la estrategia de personal y gestión de las AA.PP, para evidenciar cómo las ideas importan, han calado y se han concretado en una amalgama de garantías jurídicas que, a su vez, suponen un primer paso en la consolidación de administraciones más responsables, diversas e inclusivas. En el siguiente capítulo, quedará patente como el interés por proteger a las PCD y erradicar las circunstancias discriminatorias que impiden garantizar la igualdad de oportunidades en el empleo es universal. A continuación, se expone la acción pública efectivamente implementada en medidas para el acceso al empleo público de las PCD y el papel significativo de las AA.PP como institución fundamental para integrar el principio de igualdad en el empleo público para finalmente proponer una serie de recomendaciones dirigidas a mejorar la actuación práctica de tales instituciones.

Es el momento, por tanto, de revisar el grado de implementación de la normativa y de la acción pública, en definitiva, de identificar, cómo y hasta qué punto se está garantizando la inclusión, el acceso y promoción en el empleo público de las mujeres con discapacidad (MCD).

\section{ADMINISTRACIONES PÚBLICAS INCLUSIVAS: ALGUNOS DEBATES TEÓRICOS}

El creciente reconocimiento de la diversidad dentro de las democracias occidentales ha dado lugar a que se preste especial atención a la representatividad del personal dentro de las organizaciones públicas. Sin embargo, se han realizado pocas investigaciones sistemáticas sobre la diversidad laboral, en comparación con otros tópicos sobre organizaciones públicas (Haveman y Wolfe, 2000, p. 1012; Hahn, 1993; Rice, 2005). Solamente un $11 \%$ de los artículos publicados en revistas relevantes para la AA.PP abordan algún tema de diversidad -los dedicados a discapacidad son testimoniales- (Carrizales y Gaynor, 2013, pp. 309, 322). No obstante, se hace ineludible investigar sobre el tema en cuestión, a partir del compromiso de reducir la desigualdad previsto en la Agenda 2030 de la ONU a través de los Objetivos de Desarrollo Sostenible (ODS) tales como el 5 (igualdad de género y empoderamiento de mujeres y niñas), el 8 (crecimiento econó-

3 Sobre el estudio de este asunto insisten también otros autores como Jiménez y Huete, 2010, p. 139, Ferreira, 2008. 
mico inclusivo) y el 10 (reducción de las desigualdades), documentando las distintas fases en la consecución de la estrategia de consolidar AA.PP más inclusivas. En este trabajo comprobaremos cómo, a pesar de gran esfuerzo, España se encuentra en los estadios iniciales del proceso de consolidación de una administración conscientemente diversa.

Basándose en los trabajos de Thomas y Ely (1996) para el ámbito privado, Selden y Selden (2001) hacen una transposición de los paradigmas de diversidad en las AA.PP que pueden servir para clasificar las medidas adoptadas. Se trata de un continuum hacia organizaciones públicas totalmente integradoras, (llamadas multiculturales o integradoras de valores) en un recorrido donde se van sumando el paradigma "discriminación y justicia» (donde se prima la igual posibilidad para conseguir un empleo público, cumpliendo con las normas y acciones afirmativas); el paradigma «acceso y legitimidad» (enfatizando el valor de la diversidad para proveer de mejores servicios a cada grupo diverso) y el paradigma del «aprendizaje y efectividad» (entendiendo las implicaciones sobre el rendimiento de la organización, la diversidad de ideas, creatividad y flexibilidad en organizaciones más y mejor adaptadas). En nuestro caso, las medidas concretadas, se centran esencialmente en las dimensiones de equidad, acceso y legitimidad de la población con discapacidad en el empleo público, aún con éxito relativo.

\subsection{Administraciones públicas responsables y diversas}

Las diferentes teorías de la justicia han descuidado la atención al «acoplamiento de desventajas» que acompañan a las PCD, lo que «ha tendido a sesgar las políticas prácticas en la dirección de la inacción, e incluso ha contribuido a (...) la falta de visión responsable de la obligación social con los discapacitados» (Sen, 1999, p. 99; Sen, 2004, p. 1). De aquí el papel ejemplarizante del sector público, pues en «la medida en que el gobierno demuestra estos valores a través de sus propias prácticas de empleo, crea un modelo y un impulso para prácticas laborales similares en toda la sociedad» (Riccucci y Van Ryzin, 2017, p. 22).

Siguiendo esta lógica, a partir de la teoría de la burocracia representativa (Andrews et al., 2006, p. 291; Fredrickson, 1990; Kingsley, 1944), las organizaciones públicas deberían contar con una fuerza laboral integrada de alguna forma por los diferentes grupos que conforman la sociedad en una política de representatividad con tres pilares: «representación pasiva», promocionando la presencia de los distintos grupos sociales ${ }^{4}$; "representación activa», de impulso de los derechos e intereses de estos grupos específicos "para asegurar que los intereses de las minorías no sean ignorados en circunstancias en las que esos intereses necesitan ser escuchados y de otro modo podrían ser desoídos» (Selden et al., 1998, p. 739) y «representación simbólica», en la búsqueda de promover mayores ratios de confianza y legitimidad entre la ciudadanía, con independencia de sus características, lo que promueve una mayor legitimidad y satisfacción psicológica en el gobierno y sus servicios (Riccucci y Van Ryzin, 2017, pp. 25-28). La representatividad se contempla como un imperativo democrático con valor en sí mismo, que hace mejores administraciones, aprovechando todo el talento diverso a su disposición.

Las administraciones y las agencias del sector público, por tanto, deben de proporcionar un entorno propicio para la responsabilidad social en sentido amplio: obligando, facilitando, respaldando y colaborando con el sector empresarial y el tercer sector, pero sobre todo, asegurándose que las políticas y acciones de las propias administraciones sean socialmente responsables (Fox et al., 2002). Las AA.PP «deben aplicarse a sí mismas los mismos criterios que inspiran el concepto de responsabilidad social y hacerlo, además, con un carácter de ejemplaridad» (Ministerio de Empleo y Seguridad Social, 2014, p. 22).

Aunque una parte de la literatura de la gestión pública ha adoptado este término de responsabilidad social corporativa de forma adaptada -también llamado responsabilidad pública institucional o teoría de la responsabilidad pública y ética pública (Aguado y Marín, 2020; Carroll, 2016; Bowman y West, 2014; González y Naranjo, 2015; Lasytè, 2020; Martínez Bargueño, 1997; Navarro et al., 2010; Villoria, 2015)-, lo cierto es que la nueva gobernanza pública y la responsabilidad social se pueden reconocer en el conjunto de valores y procesos que les son comunes y que debieran hacer de guía a toda organización pública (tales como la ren-

4 Esta dimensión no es baladí, pues «la mera existencia de una burocracia representada pasivamente puede mejorar en sí misma al influir en las actitudes y comportamientos de la ciudadanía, independientemente de las acciones o resultados burocráticos» (Riccucci y Van Ryzin, 2017, p. 21). En todo caso, como recientemente recuerdan Ely y Thomas (2020, p. 1), «no hay ningún respaldo de investigación para la idea de que la diversificación de la fuerza laboral mejora automáticamente el desempeño de una organización» es requerido, de forma complementaria, un liderazgo efectivo y organizaciones verdaderamente comprometidas con la inclusión (más allá de lo numérico o lo requerido por las normas). 
dición de cuentas, apertura, transparencia, eficiencia, responsabilidad, cumplimiento de normas procesales, división de poderes y participación de los interesados). En las AA.PP se espera este compromiso y espíritu de inclusión, adalid, piedra angular y ejemplo de buenas prácticas.

A su vez, el Libro blanco sobre acceso e inclusión en el empleo público de las personas con discapacidad (INAP, 2015, p. 157) establecía como meta para el 2020 alcanzar una presencia mínima del $2 \%$ de PCD en las AA.PP. Desafortunadamente, en 2019 , aún el 50,4 \% de las empresas públicas y el $49,5 \%$ de las privadas tienen un porcentaje menor del $2 \%$ de PCD en sus plantillas (INE, 2019) ${ }^{5}$, muy lejos de los ratios de Alemania y Reino Unido (con las más altas tasas a nivel comparado).

A todo lo anterior, se suma la necesaria observancia de estas cuestiones con perspectiva de género (Finkel y Arroyo, 2019). Un $6.9 \%$ de hombres y un $6.2 \%$ de las mujeres tienen alguna discapacidad en España. De las 23.826.378 españolas, 1.573.777 tiene alguna discapacidad, 130.300 en situación de dependencia (INE, 2019). Aunque las circunstancias pueden ser múltiples y las vulnerabilidades y casuísticas enormes, el acceso al empleo público de las MCD puede verse mediatizado al aglutinar problemáticas y condicionantes que las pueden convertir en sujetos más vulnerables, a los que les resulta más difícil o gravoso acceder o promocionar en él. La discriminación interseccional de las MCD ha sido parcialmente silenciada desde el feminismo pero las consecuencias y su incidencia ha sido evidente en el desarrollo vital, en la plena incorporación a la vida social y, en concreto, en el acceso al empleo público de estas mujeres (Azcona et al., 2019; Begum, 1992; Gómez Bernal, 2012; Caballero Pérez, 2018; MacCall, 2005 y Serra, 2017)6.

Por tanto, las AA.PP más justas, inclusivas e igualitarias serían aquellas que definen acciones y desarrollan medidas para solventarlo y aquellas despojadas de viejas vinculaciones de género, esto es, "(des)genderizarlas» de los regímenes de género tradicionales, sustentadas en una ética administrativa donde los valores de igualdad de género contribuyan a la igualdad efectiva y a la calidad de las organizaciones públicas (Acker, 1992; Acker, 2006; Britton, 2000; Connell, 2006, p. 845; Ruiloba y Navarro, 2020).

En definitiva, AA.PP diversas y conscientes de los efectos de las desigualdades múltiples que den respuesta al compromiso con las PCD integrando el mandato concretado en la Convención Internacional sobre los Derechos de las Personas con Discapacidad (CDPD), adoptado por la Asamblea General de la ONU y ratificado por España en 2007 que reconoce de forma expresa la difícil situación en que se encuentran las PCD que «son víctimas de múltiples o agravadas formas de discriminación por motivos de raza, color, sexo, idioma, religión, opinión política o de cualquier otra índole, origen nacional, étnico, indígena o social, patrimonio, nacimiento, edad o cualquier otra condición» [ONU, 2006, Preámbulo, apartado p)]. Además, alienta a los Estados parte a que reconozcan el derecho de las PCD a «trabajar, en igualdad de condiciones con las demás adoptando medidas pertinentes, incluida la promulgación de legislación, entre ellas (...)» y a «emplear a PCD en el sector público» [ONU, 2006, artículo 27.1.g)].

\section{EL MARCO GENERAL DE LA PROTECCIÓN DE LAS PERSONAS CON DISCAPACIDAD}

Desde la perspectiva internacional, la protección a las PCD no se aborda, en un primer momento, como una cuestión de derechos humanos, lo que no impide que el asunto en cuestión se beneficie de la aplicación del Pacto internacional de Derechos civiles y políticos y el Pacto Internacional de Derechos Económicos, Sociales y Culturales (1966), adaptándose a las necesidades y al contexto específico de la discapacidad.

En los años 70, y a partir de instrumentos de soft-law, se abandona conscientemente el criterio rehabilitador de la discapacidad por el criterio social. La Resolución 48/96 de la Asamblea General de la ONU de 20 de diciembre de 1993 sobre normas uniformes sobre la igualdad de oportunidades de las PCD, se conforma como el estándar básico legal internacional a los efectos de la adopción de programas, leyes y políticas con relación a la discapacidad.

5 En el Boletín Estadístico del personal al servicio de las AA.PP (elaborado por el Registro Central de Personal del Ministerio de Política Territorial y Función Pública) con actualizaciones en enero y julio de cada año, no se ofrecen datos sobre personas con discapacidad en las distintas AA.PP, ni su desagregación por género o tipo de discapacidad. Tampoco en la Base estatal de datos de personas con valoración del grado de discapacidad de la Subdirección general de planificación, ordenación y evaluación (2020), se indica el número de discapacitados empleados en el sector público. Lo anterior nos dificulta poder realizar un análisis cuantitativo y usar alguno de los indicadores que sobre diversity gap se cuenta en la literatura (por ejemplo, Andrews et al., 2006).

6 Es necesario, en cambio, que se haga visible, de hecho «el concepto de interseccionalidad política insta a los legisladores y activistas a reflexionar sobre la dinámica de privilegios y exclusiones que surgen cuando se pasa por alto la atención a las personas en las intersecciones de diferentes desigualdades» (Lombardo y Verloo, 2009, p. 478). 
Con la adopción de la CDPD, anteriormente mencionada, se asume de forma indubitada el fenómeno de la discapacidad como cuestión de derechos humanos en una herramienta jurídica vinculante a la hora de hacerlos valer (Palacios y Bariffi, 2007, p. 49). El acuerdo asegura el principio de no discriminación y reconoce el derecho a trabajar en igualdad de condiciones de las PCD requiriendo a los Estados la adopción de medidas para emplear a con la singularidad de otorgar a las MCD una amplia visibilidad (artículo 6), introduciendo la transversalidad de la perspectiva de género a lo largo de todo el tratado.

De modo similar, en el ámbito europeo la prohibición de cualquier discriminación por razón de la discapacidad queda abalada en el Tratado de Ámsterdam en 1997 y con la aprobación en el año 2000 de la Carta de los Derechos Fundamentales. También, la Directiva 2000/78/CE de 27 de noviembre de 2000, relativa al establecimiento de un marco general para la igualdad de trato en el empleo y la ocupación incluye entre sus destinatarios a las PCD, promoviendo para ello, en su caso, medidas positivas contra la discriminación y de todo un elenco medidas de protección para el desarrollo de la discriminación múltiple en la UE (Giménez Gluck, 2013). En 2003, con la celebración del Año Europeo de las PCD se adoptan planes ${ }^{7}$ y estrategias, destacando la Estrategia Europea sobre discapacidad 2021-2020 ${ }^{8}$, que buscan reforzar la protección de dicho colectivo.

Además, con la aprobación en 2017 del Pilar Europeo de Derechos Sociales se actualizan los estándares de protección social ofreciendo principios estructurados entre los que destaca la igualdad de oportunidades y de acceso al mercado de trabajo y la protección e inclusión social, con especial mención a las PCD.

En coherencia con todo lo anterior, la Constitución Española (CE) reconoce de forma expresa la igualdad ante la Ley y la protección específica a las PCD en el artículo 49, ofreciendo, por un lado, cobertura suficiente para llevar a cabo políticas activas de integración -al ordenar a los poderes públicos que presten la atención especializada- y, por otro, el amparo especial necesario para el disfrute de los derechos que el Título primero otorga a todos los ciudadanos. Varias leyes se centran en la lucha contra la discriminación y la accesibilidad universal conformándose como significativos instrumentos normativos que atienden a las necesidades de las PCD; así la Ley 13/1982 de 7 de abril de integración social de las PCD (LISMI) dirigida a regular la atención y los apoyos a las PCD y sus familias -hace referencia expresa al principio constitucional de igualdad en el acceso al empleo público de las PCD $^{9}$-, la Ley 51/2003 de 2 de diciembre de igualdad de oportunidades, no discriminación y accesibilidad universal de las PCD (LIONDAU) y la Ley 39/2006 de 14 de diciembre de Promoción de la Autonomía Personal y atención a las personas en situación de dependencia (LAPAD). Las PCD se consideran como sujetos titulares de derechos; exigiendo, por ello, a los poderes públicos que garanticen el ejercicio de sus derechos de forma plena y efectiva. Las políticas públicas se reorientan dirigiendo sus objetivos a lograr la accesibilidad universal, proporcionando los apoyos necesarios para alcanzarla con criterios de equidad y sostenibilidad a fin de avanzar hacia la vida independiente y hacia una sociedad plenamente inclusiva.

\section{LA ACCIÓN PÚBLICA DISEÑADA PARA EL ACCESO DE LAS PERSONAS CON DISCAPACIDAD AL EMPLEO PÚBLICO}

La Constitución Española consagró el derecho de la ciudadanía al acceso, en condiciones de igualdad, a las funciones y cargos públicos, con los requisitos que señalen las leyes (artículo 23.2) y con los criterios de mérito y capacidad para el acceso a la función pública (artículo 103.3). La doctrina y jurisprudencia no han dudado en manifestar la conexión directa entre ambos preceptos. Su necesaria relación recíproca permite entender que el artículo 23.2 impone la obligación de no exigir para el acceso a la función pública requisito o condición alguna que no sea referible a los conceptos de mérito y capacidad

\footnotetext{
7 Plan de acción del Consejo de Europa para la promoción de los derechos y de la plena participación de las PCD en la sociedad: mejorar la calidad de vida de las PCD en Europa 2006-2015.

8 Ha sido objeto de críticas por no haberse efectuado cambios relevantes a escala europea en favor de la inclusión real de las PCD, por la falta de un enfoque de género y por la escasa apuesta por la promoción de la autonomía personal y la vida independiente, manteniéndose todavía en las políticas europeas un carácter más rehabilitador que de igualdad de derechos y oportunidades.

9 Recoge en su artículo 38 el principio constitucional de igualdad, al disponer que «en las pruebas selectivas para el ingreso en los Cuerpos de la Administración del Estado, CC.AA, Administración Local, Institucional y de la Seguridad Social serán admitidos los minusválidos en igualdad de condiciones con los demás aspirantes» (párrafo 3) y el denominado «cupo de reserva» para la contratación laboral de trabajadores y trabajadoras con discapacidad, que vinculaba tanto a las empresas públicas como a las privadas que contaran con un tamaño de 50 o más efectivos (párrafo 1).
} 
(Arroyo Yanes, 2016; Parada y Fuentetaja, 2019; Sánchez, 2020; Palomar, 2016). Como ha constatado de forma reiterada el Tribunal Constitucional, el derecho reconocido en el artículo 23.2 es una especificación del principio de igualdad del artículo $14^{10}$, que entra en juego cuando no sean aplicables ninguna de las circunstancias específicas de discriminación como raza, sexo, nacimiento, entre otras. En el ámbito de la función pública el artículo 23.2 queda como cláusula general que prohíbe toda discriminación en el acceso a funciones públicas.

Lo cierto es que, con carácter general, hasta la aprobación de nuestro texto constitucional no encontramos previsiones respecto del acceso de las $\mathrm{PCD}$ al empleo público ${ }^{11}$. A partir de ese momento, la regulación ha deambulado en normas insertadas, unas veces, en la normativa general de protección de la discapacidad y otras, en las normas propias de función pública. Si bien en un primer momento, la mayor parte de la regulación del empleo público de este colectivo se encontraba en la norma reguladora de la discapacidad (LISMI) con alguna breve referencia en normas reguladoras del empleo público, actualmente la normativa general de la función pública asume la regulación del acceso y la permanencia en el empleo de las PCD en las distintas AA.PP.

La Ley 30/1984, de 2 de agosto, de Medidas de las Reformas de la función pública -LMRFP- ha constituido el marco general del empleo público durante un largo período de tiempo, no encontrándose referencias específicas en lo relativo a la discapacidad, salvo una regulación concreta al establecer un cupo de vacantes (2\%) a raíz de la modificación efectuada por la Ley 23/1988 de 28 de julio (Disposición Adicional 19) ${ }^{12}$.

Con la incorporación de la Directiva 2000/78/CE al derecho español se afianza la apuesta por el empleo de las PCD en el sector público estableciendo el legislador de forma expresa en el TRLGDPD, el cual refunde la LISMI y la LIONDAU, que las AAPP protegerán de manera intensa los derechos de las PCD en materia de empleo, con especial atención a las personas o grupo de personas especialmente vulnerables a la discriminación múltiple como las MCD (artículo 7).

También impulsa el legislador medidas correctoras de las desigualdades de las PCD en el sector público como queda constatado en la Ley 53/2003, de 10 de diciembre, sobre empleo público de PCD, aumentando el cupo de reserva a un $5 \%$. Su desarrollo reglamentario mediante Real Decreto 2271/2004, de 3 de diciembre para la regulación del acceso al empleo público y la provisión de puestos de trabajo de las PCD, ha adquirido especial relevancia al posibilitar el fomento de acciones positivas que favorecen la igualdad de oportunidades, sin perjuicio de la igualdad de condiciones de acceso que debe imperar entre los candidatos y candidatas a la cobertura de puestos de trabajo en el sector público ${ }^{13}$.

Actualmente, la norma de referencia, el Real Decreto Legislativo 5/2015, de 30 de octubre, por el que se aprueba el Texto Refundido de la Ley del Estatuto Básico del Empleado Público (TREBEP), regula los principios rectores en la materia y redunda en constatar los principios de igualdad, de mérito y capacidad (artículo 55). Curiosamente, sin hacer mención al derecho de igualdad de oportunidades de las PCD en el acceso al empleo público, regula las medidas de acción positiva, como las cuotas de reserva de empleo en las convocatorias públicas (artículo 59).

De forma paralela, las diferentes Comunidades Autónomas (CCAA), en el ámbito de sus competencias, han reglado cuestiones relativas a las PCD en materia de asistencia social, de procedimiento para reconocimiento de la situación de dependencia, calificación de la discapacidad y el derecho de acceso a los servicios y prestaciones económicas de servicios sociales, si bien son pocas las que han dictado leyes generales reguladoras de los derechos de las PCD.

10 STC 75/1983, STC 50/1986, STC 86/1987, STC 67/1989, STC 71/1989, STC 27/1991, STC 73/1998, STC 167/1998, STC $235 / 2000$, STC 138/2000, STC 30/2008.

11 La presencia de la discapacidad ha sido mínima en la normativa de empleo público más allá de algunas normas que, tras la guerra civil, favorecieron el empleo público de los mutilados de guerra, como Decreto de 5 de abril de 1938, que aprueba el reglamento del Benemérito Cuerpo de Mutilados de Guerra por la Patria y que, entre otras medidas, favorece el acceso al empleo público de los miembros de este Cuerpo llegando a reservar el $30 \%$ de las plazas en importantes cuerpos de la Administración, tanto estatal como local y en las empresas públicas y la Ley 5/1976, de 11 de marzo, de Mutilados de Guerra por la Patria. El Decreto 2351/1970, de 22 de agosto, sobre empleo de trabajadores minusválidos, establece, con carácter general, las condiciones especiales de acceso al empleo de las PCD.

12 Dictada como consecuencia de la STC de 11 de junio de 1987 que declara inconstitucionales algunos artículos de la Ley 30/1984.

13 Un precedente del mismo lo encontramos en el Reglamento General de Ingreso del personal al servicio de la Administración General de Estado y de Provisión de puestos de trabajo y promoción profesional de los Funcionarios Civiles de la Administración General del Estado (AGE), aprobado por Real Decreto 364/1995, de 10 de marzo. 
Figura 1. NoRmativa SOBRE LA PROTECCIÓn DE PCD y ACCESO A LA FUnCIÓn PÚBLICA NORMATIVA RELATIVA A LA PROTECCIÓN DE PERSONAS DISCAPACITADAS Y ACCESO AL EMPLEO PÚBLICO

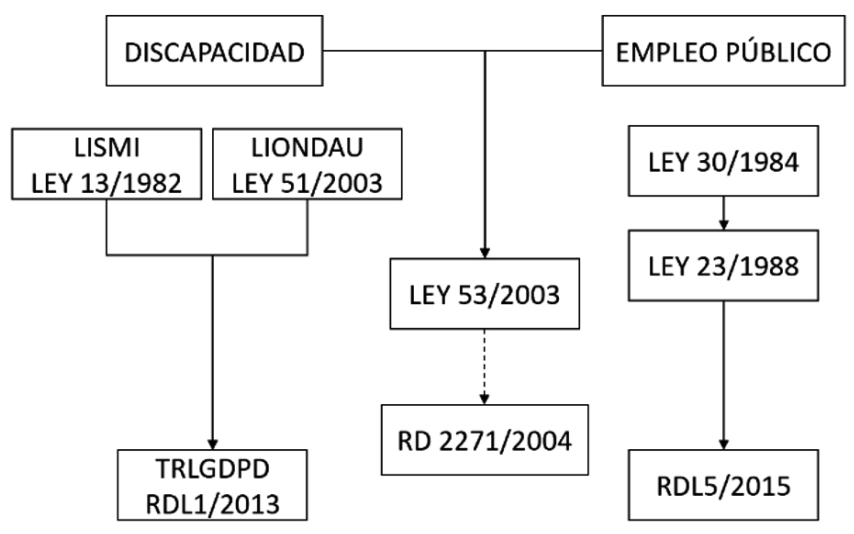

Fuente: Elaboración propia.

\subsection{Medidas de acción positiva para promover el acceso al empleo público}

Las cuotas de reserva de empleo en las convocatorias públicas son una excepción al principio de mérito y capacidad a favor de las PCD que se extiende al diseño de los procesos selectivos mediante ajustes razonables de tiempo y medios que faciliten su participación, así como la adaptación de los puestos de trabajo a sus necesidades.

Con carácter general la LISMI establecía la cuota de reserva de empleo público fijando un marco jurídico específico para tratar la problemática de las PCD, pero es la Ley 23/1988, de 28 de julio la que establece la necesidad de recoger en las ofertas de empleo público un porcentaje obligatorio de cupo de plazas para el colectivo en cuestión ${ }^{14}$. Seguidamente la Ley 53/2003, eleva el cupo al $5 \%$ y en las ofertas de empleo público del 2009, aprobada por Real Decreto 248/2009, de 27 de febrero, y 2010, aprobada por Real Decreto 406/2010, de 31 de marzo, se amplió el porcentaje al 7\%, tal como se establecía en la Ley 7/2007 de 12 de abril, del Estatuto Básico del Empleado Público (EBEP).

Actualmente, el artículo 59 del TREBEP establece que «en las ofertas de empleo público se reservará un cupo no inferior al $7 \%$ de las vacantes para ser cubiertas entre PCD»; de éste $7 \%$, un $2 \%$ se destina a PCD intelectual. Ésta es la norma básica, aplicable a todos los procesos selectivos, pero las AAPP territoriales (CC.AA) pueden elevar este cupo y adoptar sus propias normas más favorables sobre las adaptaciones y ajustes razonables de tiempos y medios en el proceso selectivo y, una vez superado dicho proceso, las adaptaciones en el puesto de trabajo a sus necesidades. La legislación autonómica, anterior y posterior al EBEP, también ha recogido cupos de reserva para el acceso a la función pública de PCD. Los porcentajes mínimos y máximos de reserva han oscilado entre el $3 \%$ de Aragón y Cataluña; el $6 \%$ de Madrid; el $7 \%$ de Andalucía, Galicia y Castilla La Mancha; y el $10 \%$ de Extremadura y Castilla y León, entre otras.

De forma particular, son interesantes las objeciones y reflexiones planteadas por parte de la doctrina en relación al devenir de los acontecimientos del modelo español de la inclusión de cupo o reserva de plazas vía porcentaje en el empleo funcionarial (Arroyo, 2016). Por una parte, dado el transcurso de amplios lapsos de tiempo sin publicación de oferta de empleo público, se aconsejaría la búsqueda de mecanismos para exigir a las organizaciones públicas realizar tales ofertas y conseguir que quienes tienen capacidades diversas consigan una mayor cuota de penetración. Por otra parte, se debería replantear el cupo o reserva aportando nuevas variables a tener en cuenta, entre otras, el número de PCD que han sido ya reclutadas y el porcentaje que cabe establecer de ellas respecto de todo el conjunto de esa organización; las plazas dotadas presupuestariamente más ajustadas a este tipo de reclutamiento y el porcentaje total de PCD dentro

14 Introduce la primera cuota de reserva añadiendo la Disposición Adicional 19 a la Ley 30/1984 en un $3 \%$ y relegando a un momento posterior al desarrollo de la oferta, el proceso de adaptación funcional. 
del ámbito territorial de actuación de la AAPP que, encontrándose en edad de trabajar en ella (desde los 16 años hasta la edad prevista de jubilación en cada caso), se encuentran imposibilitadas de hacerlo. Por último, se debería reflexionar sobre los problemas de incumplimiento de las cuotas ya señalados, la necesidad de soluciones más firmes, o medidas que podrían ser efectivas, como la imposición a las empresas privadas -vía contratación administrativa- de unos parámetros en los que se valorara especialmente la contratación laboral de PCD u otros modos como la promoción, creación y puesta en marcha de Centros especiales de empleo en las AAPP en un impulso realmente intencionado de una política pública de personal decidida en construir AAPP diversas e inclusivas.

\subsection{Otras medidas que favorecen la integración}

La normativa prevé la aplicación de otras medidas que tienen por objeto favorecer la integración de las PCD mediante: la facilidad de acceso a la formación, la preferencia en la elección de destino, alteración del orden de prelación por razones debidamente acreditadas, la adaptación de tiempos adicionales en los procesos selectivos y en los puestos de trabajo, la posibilidad de realizar cursos específicos a aquellos con especiales dificultades para integrarse en el puesto de trabajo o para promocionar en puestos ordinarios. De forma expresa, se permite que la Administración podrá realizar cursos de formación destinados únicamente a PCD (artículo 11 Decreto 2271/2004).

Recientemente el Instituto Nacional de Administración Pública (INAP) ha aprobado el I Plan de formación en igualdad y no discriminación 2020-2022, impulsando con este proyecto su interés de contribuir a hacer realidad el principio de igualdad de oportunidades, promover una Administración más plural, diversa y representativa y en el que se proponen expresamente acciones formativas de carácter básico centradas en el eje de igualdad y no discriminación referidas a la discapacidad en el entorno laboral.

Por otro lado, es indudable que las AA.PP pueden influir en la actuación de sus licitadores proveedores de productos y servicios. De este modo, la Administración, en sus relaciones con licitadores y subcontratistas, puede constituirse en referente, favoreciendo las relaciones comerciales con aquellas empresas que cumplan la normativa en materia de empleo de PCD. En la actualidad, la vigente Ley 9/2017, de 8 de noviembre, de Contratos del Sector público, por la que se transponen al ordenamiento jurídico español las Directivas del Parlamento Europeo y del Consejo 2014/23/UE y 2014/24/UE, de 26 de febrero de 2014, admite en su artículo 150 la posibilidad de que los órganos de contratación utilicen criterios sociales de valoración de las ofertas.

Es oportuno apuntar también que tanto a nivel nacional como autonómico se han creado órganos específicos de asesoramiento para el acceso al empleo público de las PCD, con competencias principales de tipo consultivo entre las que destacan, la emisión de informes de asesoramiento a todas las AAPP con competencias en el sector para la elaboración de proyectos o iniciativas normativas que afecten específicamente a la población con discapacidad y el seguimiento de las actuaciones de los poderes públicos destinadas a las PCD ${ }^{15}$.

TABLA 1. Medidas PARA LA PROMOción DEL EMPLEO PÚBlico destinAdas A LAS PCD

\begin{tabular}{ll}
\hline \multicolumn{2}{c}{ MEDIDAS DE ACCIÓN POSITIVA } \\
\hline \multicolumn{1}{c}{ CUOTAS DE RESERVA } & \multicolumn{1}{c}{ OTRAS MEDIDAS } \\
\hline LISMI (carácter general) & Cursos de formación destinados PCD \\
\hline Ley 23/1988 (carácter necesario) - 3\% & I Plan formación \\
\hline Ley 53/2003 - 5\% & Órganos de asesoramiento \\
\hline EBEP 2007 - 7\% & Consejo nacional de discapacidad \\
\hline TREBEP $2015-7 \%$ & Criterios sociales en contratación pública \\
\hline
\end{tabular}

Fuente: Elaboración propia.

15 Entre otros, el Consejo Nacional de la Discapacidad, el Consejo Andaluz de atención a las PCD, el Consejo Asesor de PCD en la Comunidad de Madrid, el Consejo Asesor regional de PCD en la Región de Murcia. 


\section{EL PAPEL DE LA ADMINISTRACIÓN EMPLEADORA Y PROMOTORA DE LA IGUALDAD DE GÉNERO}

Las AA.PP tienen un papel preponderante en la consecución de una igualdad real y efectiva en el marco de un Estado social y democrático de derecho. Tanto en el plano internacional como en el europeo la inquietud por promover políticas de igualdad de género en la esfera laboral queda patente en diferentes instrumentos jurídicos que consolidan e igualan los derechos entre géneros. Actores internacionales como la ONU con la Carta (1945), la Declaración Universal de los Derechos Humanos (1948) o la celebración de la Convención sobre la eliminación de todas las formas de discriminación contra la mujer (CEDAW) (1979) ${ }^{16}$, e instituciones comunitarias de gran peso como la Comisión Europea y el Consejo de Europa realizan una enérgica actuación con importantes repercusiones en el ámbito laboral ${ }^{17}$ que quedan constatadas, a su vez, en varios documentos como la Carta de los Derechos Fundamentales de la UE y una abundante normativa comunitaria como la jurisprudencia del propio Tribunal de Justicia de la Comunidad Europea ${ }^{18}$.

De forma particular, las AA.PP han de integrar el principio de igualdad de trato y oportunidades entre géneros de manera real y efectiva, con carácter transversal y de forma activa estableciendo medidas correctoras, como las acciones positivas, que pudiesen coadyuvar a combatir la discriminación y colaborar y auxiliarse mutuamente en orden a lograr que el acceso a la función pública sea en condiciones de igualdad, respetando a su vez los principios de mérito y capacidad (Belando, 2018, pp. 771-775). Todo ello sin descuidar su función como sujeto de control que velará porque terceros no lleven a cabo actuaciones discriminatorias por razón de género (Menéndez, 2012, pp. 54 y ss.) y favorecer, así, la justicia social.

Por tanto, el hecho que no todas las personas tienen las mismas oportunidades, requiere de una AA.PP protectora de la igualdad, vigilante y que presta especial atención a personas especialmente vulnerables a la discriminación múltiple como las MCD.

\subsection{El reto de incorporar soluciones a la discriminación múltiple en la normativa general de la función pública}

Las dificultades agravadas a las que se podrían enfrentar las MCD, han sido objeto de un mínimo tratamiento normativo en el ámbito de la función pública. La Ley Orgánica 3/2007, de 22 de marzo, para la igualdad efectiva de mujeres y hombres (LOI) al igual que el EBEP, no incorpora ninguna previsión positiva de preferencia en el acceso de las mujeres a la Función Pública; en cambio, sí apuesta por integrar aspectos relativos del principio de igualdad de género en el empleo público $(T \text { Título } V)^{19}$. Tal apuesta hemos de entenderla imprescindible para la identificación del colectivo de MCD como sujetos diana para la concreción de medidas reforzadas para garantizar la igualdad en el acceso y ascenso en el sector público. Las acciones que han visibilizado la necesidad de favorecer la perspectiva combinada de inclusión de la perspectiva de género y discapacidad han sido las anteriormente reseñadas Estrategia española sobre discapacidad 20122020 y el Plan de Acción 2014-2020. Recientemente en marzo de 2021 se ha aprobado por la Comisión

16 A ello se suman Conferencias mundiales sobre la mujer que han contribuido a situar la causa de la igualdad de género en el debate mundial. Es la segunda Conferencia (Copenhague, 1980) donde se apuesta por la igualdad de oportunidades en el empleo como línea de actuación. En la de Beijing (1995), el tratamiento de la igualdad se tiñe de la perspectiva de género adoptándose la Declaración y la Plataforma de Acción con estrategias fundamentales para el desarrollo eficaz de las políticas de igualdad como la transversalidad de género y la representación equilibrada.

17 La Comisión Europea, después de renovar su compromiso por la igualdad entre mujeres y hombres en la Carta de la Mujer (2010), aprueba, por un lado, la Estrategia «Europa 2020: una estrategia para un crecimiento inteligente, sostenible e integrador», apostando por medidas que eliminen las barreras para la participación de las mujeres en el mercado laboral y, por otro, su «Compromiso estratégico para la igualdad entre mujeres y hombres 2016-2019». En consonancia con ello, el Consejo de Europa en el II Pacto Europeo para la lgualdad de Género 2011-2020 y en el Estrategia de Igualdad de Género para 2018-2023 insta a los Estados y a la UE a adoptar medidas para cerrar las brechas de género en el empleo, promover una mejor conciliación de la vida laboral y vida privada para mujeres y hombres.

18 La jurisprudencia del TJCE evoluciona desde una primera distinción entre discriminación directa e indirecta a una discriminación por razón de sexo, con especial atención en cuanto al embarazo. Sentencia TJCE 17 Octubre 1995-asunto Kalanke y Sentencia TJCE 11 Noviembre 1997-asunto Marshall.

19 El Capítulo I regula el principio de igualdad en el empleo público estableciéndose los criterios generales de actuación a favor de la igualdad para el conjunto de las AA.PP y en su Capítulo II, la presencia equilibrada de mujeres y hombres tanto en los nombramientos de órganos directivos de la AGE como a los órganos de selección y valoración del personal. El Capítulo III se dedica a las medidas de igualdad en el empleo, con la previsión específica del mandato de aprobación de un protocolo de actuación frente al acoso sexual y por razón de sexo. 
GAPP. Nueva Época - N. ${ }^{2}$ 28, marzo 2022 - ISSN: 1989-8991 - DOI: https://doi.org/10.24965/gapp.i28.10901 - [Págs. 112-126] ¿Administraciones públicas inclusivas?: la implementación de la normativa sobre el acceso de las mujeres con discapacidad...

Rocío Navarro González / Juana María Ruiloba Núñez

Europea la Estrategia de las PCD 2021-2030 que tendrá repercusión, de una manera u otra, en nuestra acción pública nacional.

\subsection{Los Planes de Igualdad en las Administraciones Públicas}

La entrada en vigor de la LOI facilita la regulación de la discapacidad en el empleo público con dimensión de género. Ha supuesto el impulso en la planificación de acciones a favor de la igualdad real plasmadas en Planes estratégicos de igualdad de oportunidades. El vigente III Plan Estratégico de Igualdad de Oportunidades -al igual que ya ocurría en el II-, prevé en uno de sus ejes de actuación -el relativo a la igualdad de género en el ámbito laboral-, la apuesta por la adopción de medidas que promuevan la igualdad de trato y oportunidades en el empleo del sector público. No obstante, no es hasta el III Plan de igualdad de género de la AGE y en sus organismos públicos, aprobado recientemente ${ }^{20}$, cuando se introduce en el sexto eje denominado «interseccionalidad y situaciones de especial protección», el objetivo expreso de superar la discriminación relacionada con la discapacidad y la diversidad funcional, con perspectiva de género.

Como muestra de ello, en dicho Plan se recogen medidas relativas a diagnosticar con perspectiva de género la situación actual de los empleados y empleadas públicas con discapacidad en la AGE, identificando las líneas de actuación ya implantadas con éxito para suprimir la discriminación de las PCD con perspectiva de género y evaluar sus resultados. A ello se suman medidas para formar y sensibilizar sobre discapacidad dentro de la AGE y empoderar a las MCD y tratar la discapacidad como situación de especial protección con perspectiva de género o promover la investigación comparada y el estudio (Benchmarking) de «buenas prácticas en políticas de igualdad de mujeres y hombres con discapacidad en Administraciones Públicas de países de referencia».

\section{RECOMENDACIONES PARA MEJORES PRÁCTICAS EN LAS AA.PP}

En esta nueva fase de planificación de la política pública sobre discapacidad que arranca en 2021 con nuevas estrategias a nivel comunitario y nacional muchos son los desafíos a los que hacer frente ${ }^{21}$ : «las barreras que hacen que no se cubra la reserva del $7 \%$ para las PCD; su presencia en las comisiones de selección; el seguimiento de su carrera una vez que han accedido a la Administración; la adecuación de los procesos selectivos actuales para atraer más talento, no solo de las PCD...» o la misma consideración de lo que la discapacidad es y cómo es tenida en cuenta en las acciones y políticas públicas diseñadas (INAP, 2015, p. 9), planteándose las acciones positivas como objetivo principal (Azkona et al., 2019).

Pues, aunque se han redoblado esfuerzos para facilitar la participación en condiciones de igualdad de las PCD, las adaptaciones contenidas en el Real Decreto 2271/2004, de 3 de diciembre, por el que se regula el acceso al empleo público y la provisión de puestos de trabajo de las PCD, «parecen insuficientes no solo para el acceso al proceso selectivo, sino también para cubrir las plazas reservadas. Ello hace pensar que serían necesarias no solo adaptaciones de tiempos y/o medios, sino adaptaciones referidas al tipo de proceso selectivo para cubrir las plazas convocadas» (INAP, 2015, p. 59), si añadimos la dimensión género, más limitaciones se añaden aún.

A finales de marzo de 2019, el Comité de la ONU sobre los derechos de las personas con discapacidad (CRPD), en el seguimiento a nivel internacional de la labor de cada Estado parte, aprobó una serie de observaciones finales en su sesión $463 .^{\circ}$ en las que ha constatado su preocupación acerca de las formas múltiples de discriminación a las que se enfrentan las MCD. De forma particular, sugiere al Estado español una serie de recomendaciones relativas a adoptar medidas eficaces y específicas que garanticen la igualdad y prevengan las formas múltiples e interseccionales de discriminación de las mujeres y niñas con discapaci-

20 Resolución de 29 de diciembre de 2020, de la Secretaría General de Función Pública por la que se publica el Acuerdo de Consejo de Ministros de 9 de diciembre de 2020, por el que se aprueba el III Plan para la igualdad de género en la AGE y en los Organismos Públicos vinculados o dependientes de ella.

21 Cuando se indaga sobre los motivos, en estudios como el de Guillem y Contreras (2010, p. 99), el 94\% de los gestores públicos encuestados declara que la razón de no tener contratadas a personas discapacitadas es no haber tenido candidatos en los procesos de selección del personal (en el $57,8 \%$ de los casos no se presentó ninguna persona discapacitada a las convocatorias -esencialmente oposiciones-, o no han recibido currículos, según el 36,2\%). 
dad en sus políticas de igualdad de género, e incorpore de manera transversal la perspectiva de género en su legislación y sus políticas en materia de discapacidad ${ }^{22}$.

También sugiere el Comité que, de conformidad con la meta 8.5 de los Objetivos de Desarrollo Sostenible, analice y modifique la legislación española, los reglamentos y las políticas, a fin de promover el empleo de las PCD en los sectores público y privado, con especial atención a las mujeres y a aquellos y aquellas que residen en zonas rurales.

Por otra parte, resultaría enriquecedor -como hemos dejado apuntado en líneas anteriores a raíz de iniciativas doctrinales- la flexibilización de las bolsas de trabajo, la búsqueda de mecanismos para exigir a las organizaciones públicas realizar tales ofertas ante el transcurso de lapsos de tiempo prolongados sin publicarse y replantear el cupo o reserva aportando nuevas variables a tener en cuenta, entre otras, el número de PCD que han sido ya reclutadas o el porcentaje que cabe establecer de ellas respecto de todo el conjunto de esa organización o departamento concreto o las cuotas de género en el caso de clara infrarrepresentación de las mujeres con discapacidad.

A su vez, sería interesante evaluar la idoneidad de trasponer y adaptar los modelos de acceso e inclusión de las personas con discapacidad en el empleo público adoptados por parte de otros países de nuestro entorno a modo de mejoras o buenas prácticas; así, por ejemplo, tanto en Alemania como en Reino Unido se ofrece la posibilidad de realizar, de manera añadida, entrevistas personales en las que delimitar mejor las competencias y habilidades que el candidato o candidata puedan desempeñar. En concreto, en Alemania, una vez realizada la selección, se propone la firma de acuerdos de integración en la que se establezcan la carrera y el ascenso dentro del sector público. Un mecanismo que no solo se enfoca en el acceso sino en la propia carrera de los y las empleadas públicas con discapacidad.

En otro orden de consideraciones, la apuesta por el teletrabajo en la situación de pandemia en la función pública y el tránsito hacia modelos de empleo público híbridos genera la obligatoriedad de replantearse nuevos medios y materiales para su ejercicio por parte de las personas con discapacidad -haciendo frente a los posibles obstáculos del trabajo en remoto-.

Finalmente, sería recomendable que la Administración invirtiera en investigaciones y proyectos manteniendo presente el interés por un continuo estudio y análisis de la cuestión objeto de análisis, el acceso e integración efectiva al empleo público de las personas con discapacidad, sin olvidar la dimensión de género. Son importantes las reflexiones para reformular las prácticas desde las propias AA.PP como el Taller que durante los meses de junio y julio de este año 2021 se ha organizado sobre Medidas para favorecer el acceso al empleo público de las personas con discapacidad y que implica no solo a la Administración pública española sino también a los gobiernos iberoamericanos ${ }^{23}$.

\section{CONCLUSIONES}

La apuesta creciente por medidas que favorezcan el acceso al empleo público de las PCD es significativa y, pese a que la protección de dicho colectivo tiene respaldo normativo, no es suficiente para garantizar una igualdad plena ni prevenir totalmente las formas de discriminación múltiple a las que se vienen enfrentando las mujeres con discapacidad.

Tras el recorrido teórico, por la normativa de referencia en el ámbito internacional, europeo y nacional, por las acciones públicas diseñadas y los límites de su implementación expuestas en las páginas anteriores, podemos concluir que urge el apuntalamiento de AA.PP más responsables e igualitarias, que tengan como bandera la diversidad y la incorporación de la perspectiva de género en la función pública dando un giro a las políticas públicas de empleo en el sector público hacia estadios más avanzados en la inclusión y normalización de la presencia de las PCD, especialmente de las mujeres. La puesta en marcha de los trámites necesarios para la elaboración de la Estrategia Española sobre discapacidad 2021-2030 es una buena oportunidad para seguir avanzado en ello.

22 Observaciones finales sobre los informes periódicos segundo y tercero combinados de España del Comité sobre los Derechos de las Personas con Discapacidad, 9 de abril de 2019.

23 Marco del Plan de Transferencia, intercambio y gestión de conocimiento para el desarrollo de la cooperación Española en América Latina y el Caribe -INTERCOONECTA-, el Instituto Nacional de Administración Pública (INAP) en colaboración con la Organización Iberoamericana de Seguridad Social (OISS), el Grupo Social ONCE, en calidad de Unidad Técnica del Programa Iberoamericano de Discapacidad (PID) y la AECID. 


\section{REFERENCIAS BIBLIOGRÁFICAS}

Abberley, P. (1998). Trabajo, utopía e insuficiencia. En L. Barton (coord.), Discapacidad y sociedad, Morata.

Acker, J. (1992). From sex roles to gendered institutions. Contemporary Sociology, 21(5), 565-569. https://doi. org/10.2307/2075528

Acker, J. (2006). Inequality regimes gender, class, and race in organizations. Gender and Society, 20(4), 441-464. https://doi.org/10.1177/0891243206289499

Andrews, R., Boyne, G. A. y Walker, R. M. (2006). Workforce diversity in the public sector: an evaluation of the performance of English local authorities. Policy and Politics, 34(2), 287-306. http://dx.doi. org/10.1332/030557306776315868

Aguado Hernández, J. A. y Marín Traura, S. (2020). La inclusión laboral de las personas con discapacidad desde la Administración Pública responsable en la Comunidad Valenciana. Siglo Cero Revista Española Sobre Discapacidad Intelectual, 51(3), 7-26. https://doi.org/10.14201/scero2020513726

Arroyo Yanes, L. M. (2016). Los instrumentos de gestión del empleo público. INAP.

Azkona, N., Rekakoetxea, Z. y García, A. (2019). Diagnóstico de la situación laboral de las mujeres con diversidad funcional de la CAE. Vitoria-Gasteiz: Emakunde Instituto vasco de la Mujer. https://www.euskadi.eus/contenidos/ informacion/publicaciones_bekak/es_def/adjuntos/beca.2018.2.genero_edad_df_empleo.pdf

Begum, N. (1992). Disabled women and the feminist agenda. Feminist $\bar{R}$ eview, 40(1), 70-84. https://doi. org/10.2307/1395278

Belando Garin, B. (2018). Igualdad efectiva en la Administración Pública. En El derecho a la igualdad efectiva de mujeres y hombres: una evaluación del primer decenio de la LO 3/2007 (pp. 771-775), Aranzadi.

Bowman, J. y West, J. P. (2014). Public service ethics: Individual and Institutional Responsibilities. Sage.

Britton, D. (2000). The epistemology of the gendered organizations. Gender and Society, 14(3), 418-434. https://doi. org/10.1177/089124300014003004

Caballero Pérez, I. (2018). Feminism and disability: The human right to inclusive education from a gender perspective. Journal of Feminist, Gender and Women Studies, 7, 5-13. https://doi.org/10.15366/jfgws2018.7.001

Carroll, A. B. (2016). Carroll's pyramid of CSR: taking another look. International Journal of Corporate Social Responsibility, 1, article 3. https://doi.org/10.1186/s40991-016-0004-6

Carrizales, T. y Gaynor, T. S. (2013). Diversity in public administration research: A review of journal publications. Public Administration Quarterly, 37(3), 306-330. https://www.jstor.org/stable/24372110

Comisión Europea (2007). Tackling multiple discrimination. Oficina de Publicaciones de la UE. https://op.europa.eu/en/ publication-detail/-/publication/f1f6da3a-2c36-4ef7-a7c2-b906349220b4

Connell, R. (2006). Glass Ceilings or gendered institutions? Mapping the gender regimes of public sector worksites. Public Administration Review, 66(6), 837-849. https://doi.org/10.1111/j.1540-6210.2006.00652.x

Conner, T. W. (2016). Representation and Collaboration: Exploring the Role of Shared Identity in the Collaborative Process. Public Administration Review, 76(2), 288-301. https://doi.org/10.1111/puar.12413

Grant, M. J. y Booth, A. (2009). A typology of reviews: analysis of 14 review types and associated methodologies. Health Information and Libraries Journal, 26(2), 91-108. https://doi.org/10.1111/j.1471-1842.2009.00848.x

Guillén, C. y Contreras, R. (2010). Discapacitados y empresas. Un análisis de la productividad. El coste de oportunidad desconocido. Fundación Mapfre. https://sid-inico.usal.es/documentacion/discapacitados-y-empresas-un-analisisde-la-productividad-el-coste-de-oportunidad-desconocido/

Ely, R. J. y Thomas, D. A. (2020). Getting Serious About Diversity: Enough Already with the Business Case. Harvard Business Review [artículo digital]. https://hbr.org/2020/11/getting-serious-about-diversity-enough-already-with-thebusiness-case

Ferreira, M. A. V. (2008). Una aproximación sociológica a la discapacidad desde el modelo social: apuntes caracteriológicos. REIS. Revista Española de Investigaciones Sociológicas, 124, 141-174. http://www.reis.cis.es/ REIS/jsp/REIS.jsp?opcion=articulo\&ktitulo=2158\&autor=MIGUEL+A.+V.+FERREIRA

Fredrickson, H. G. (1990). Public administration and social equity. Public Administration Review, 50(2), 228-237. https:// doi.org/10.2307/976870

Finkel, L. y Arroyo, M. (coords.) (2019). Mujer con discapacidad y enfermedades crónicas: género, discapacidad y cronicidad. Informe de resultados. Observatorio Estatal de la Discapacidad. https://www. observatoriodeladiscapacidad.info/mujer-con-discapacidad-y-enfermedades-cronicas-genero-discapacidad-ycronicidad/

Fox, T., Ward, H. y Howard, B. (2002). Public sector roles in strengthening corporate social responsibility: a baseline study. International Institute for Environment and Development. https://pubs.iied.org/16017iied

Gómez Bernal, V. (2012). Procesos, significados y efectos en torno a la catalogación oficial de las discapacidades en las mujeres. Nómadas: Critical Journal of Social and Juridical Sciences, 35(3), 225-266. https://doi.org/10.5209/ rev_NOMA.2012.v35.n3.42204

Giménezz Gluck, D. (2013). La discriminación múltiple en el Derecho de la Unión. Revista Española de Derecho Europeo, 45, 113-136. http://www.revistasmarcialpons.es/revistaespanoladerechoeuropeo/article/view/400discirminacion-multiple-derecho-union 
GAPP. Nueva Época - N. ${ }^{\circ}$ 28, marzo 2022 - ISSN: 1989-8991 - DOI: https://doi.org/10.24965/gapp.i28.10901 - [Págs. 112-126]

¿Administraciones públicas inclusivas?: la implementación de la normativa sobre el acceso de las mujeres con discapacidad..

Rocío Navarro González / Juana María Ruiloba Núñez

González Soleto, C. y Naranjo Africano, G. (2015). La responsabilidad institucional pública: un análisis para el mejoramiento de la gestión y la gerencia en la administración pública. Revista CIFE: Lecturas de Economía Social, 17(27), 245-296. https://doi.org/10.15332/s0124-3551.2015.0027.07

Hahn, H. (1985). Towards a Politics of Disability: Definitions, Disciplines, and Policies. Independent Living Institute (consultado el 16-12-2020). http://www.independentliving.org/docs4/hahn2.html

Hahn, H. (1993). The Political Implications of Disability Definitions and Data. Journal of Disability Policy Studies, 4(2), 41-52. https://doi.org/10.1177/104420739300400203

Haveman, R. y Wolfe, B. (2000). The economics of disability and disability policy. En A. J. Culyer y J. P. Newhouse (eds.), Handbook of Health Economics (vol. 1B, pp. 995-1051). Elsevier Science.

Instituto Nacional de Administración Pública - INAP (2015). Libro blanco sobre acceso e inclusión en el empleo público de las personas con discapacidad. INAP.

Instituto Nacional de Estadística - INE (2019). El Empleo de las Personas con Discapacidad. Serie 2014-2019. https://www.ine.es/dyngs/INEbase/es/operacion.htm?c=Estadistica_C\&cid=1254736055502\&menu=resultados\&i $\mathrm{dp}=1254735976595$

Jiménez Lara, A. y Huete García, A. (2010). Políticas Públicas sobre discapacidad en España. Hacia una perspectiva basada en los derechos. Política y Sociedad, 47(1), 137-152. https://revistas.ucm.es/index.php/POSO/article/view/ POSO1010130137A

Kingsley, J. D. (1944). Representative Bureaucracy: An Interpretation of the British Civil Service. Antioch Press.

Lasytè, G. (2020). Socially Responsible Organisational Governance in the Public Sector. Public Policy and Administration, 19(3), 119-132. http://dx.doi.org/10.5755/j01.ppaa.19.3.27713

Llewellyn, A. y Hogan, K. (2000). The Use and Abuse of Models of Disability. Disability \& Society, 15(1), 157-165. https://doi.org/10.1080/09687590025829

Lombardo, E. y Verloo, M. (2009). Institutionalizing Intersectionality in the European Union? International Feminist Journal of Politics, 11(4), 478-495. https://doi.org/10.1080/14616740903237442

McCall, L. (2005). The complexity of intersectionality. Signs: Journal of Women in Culture and Society, 30(3), 17711800. https://doi.org/10.1086/426800

Makkonen, T. (2002). Múltiple, Compound and Intersectional Discrimination: bringing the experiences of the most marginalized to the fore. Institute for Human Rights, Abo Akademi University. https://www.abo.fi/wp-content/ uploads/2018/03/2002-Makkonen-Multiple-compound-and-intersectional-discrimination.pdf

Martínez Bargueño, M. (1997). La ética, nuevo objetivo de la gestión pública. Gestión y Análisis de Políticas Públicas, 10, 19-32. https://doi.org/10.24965/gapp.vi10.125

Menéndez Sebastián, E. M. (coord.) (2012). La Administración promotora de la lgualdad de Género. Tirant lo Blanch.

Ministerio de Empleo y Seguridad Social (2014). Estrategia Española de Responsabilidad Social 2014-2020 (EERSE). Estrategia 2014-2020 para empresas, administraciones públicas y el resto de organizaciones para avanzar hacia una sociedad y una economía más competitiva, productiva, sostenible e integradora. https://www.mites.gob.es/es/ rse/eerse/index.htm

Navarro, A., Alcaráz, F. J. y Ortíz, D. (2010). La divulgación de información sobre responsabilidad corporativa en administraciones públicas: un estudio empírico en gobiernos locales. Revista de Contabilidad, 13(2), 285-314. https://doi.org/10.1016/S1138-4891(10)70019-4

Organización de Naciones Unidas (ONU) (2006). Convención Internacional sobre los Derechos de las Personas con Discapacidad. https://www.ohchr.org/sp/hrbodies/crpd/pages/disabilitiesconvention.aspx

Organización de Naciones Unidas (ONU) (2019). Estrategia de las Naciones Unidas para las personas con discapacidad. https://www.un.org/es/content/disabilitystrategy/

Onwuegbuzie, A. y Frels, R. (2016). Seven Steps to a Comprehensive Literature Review: A Multimodel \& Cultural Approach. Sage.

Palacios, A. y Bariffi, F. (2007). La discapacidad como una cuestión de derechos humanos. Una aproximación a la Convención Internacional sobre los derechos de las personas con discapacidad. Ediciones Cinca.

Palomar Olmeda, A. (2016). Derecho de la Función Pública: régimen jurídico de los funcionarios públicos (11. a ed.). Dykinson.

Parada Vázquez, R. y Fuentetaja Pastor, J. (2019). Derecho de la Función Pública (2. ${ }^{a}$ ed). Thomson Reuters-Civitas

Rey Martínez, F. (2008). La discriminación múltiple, una realidad antigua, un concepto nuevo. Revista Española de Derecho Constitucional, 84, 251-283. https://recyt.fecyt.es/index.php/REDCons/article/view/48137

Rice, M. F. (ed.) (2005). Diversity and Public Administration: Theory, Issues, and Perspectives. M. E. Sharpe.

Riccucci, N. M., y Van Ryzin, G. G. (2017). Representative Bureaucracy: A Lever to Enhance Social Equity, Coproduction, and Democracy. Public Administration Review, 77(1), 21-30. https://doi.org/10.1111/puar.12649

Ruiloba Núñez, J. M. y Navarro González, R. (2020). ¿Administraciones públicas «genderizadas»? Revista Derecho del Estado, 47, 343-369. https://doi.org/10.18601/01229893.n47.11

Sánchez Morón, M. (2020). Derecho de la función Pública (13. ${ }^{a}$ ed.). Tecnos.

Sen, A. (1999). Development as Freedom. Oxford University Press.

Sen, A. (2004, 30 de noviembre-1 de diciembre). Disability and justice [Discurso en la Conferencia Internacional de Discapacidad]. Disability and Inclusive Development: Sharing, Learning and Building Alliances. World Bank. 
Selden, S. C. (2006). A Solution in Search of a Problem? Discrimination, Affirmative Action, and the New Public Service. Public Administration Review, 66(6), 911-923. https://doi.org/10.1111/j.1540-6210.2006.00659.x

Selden, S. C., Brudney, J. L. y Kellough, J. E. (1998). Bureaucracy as a Representative Institution: Toward a Reconciliation of Bureaucratic Government and Democratic Theory. American Journal of Political Science, 42(3), 717-744. https://doi.org/10.2307/2991727

Selden, S. C. y Selden, F. (2001). Rethinking diversity in public organizations for the 21st century: Moving toward a multicultural model. Administration \& Society, 33(3), 303-329. https://doi.org/10.1177/00953990122019785

Serra, M. L. (2017). Mujeres con discapacidad: sobre la discriminación y opresión interseccional. Dykinson

Thomas, D. A. y Ely, R. J. (1996, septiembre-octubre). Making differences matter: A new paradigm for managing diversity. Hardvard Bussiness Review. https://hbr.org/1996/09/making-differences-matter-a-new-paradigm-formanaging-diversity

Villoria Mendieta, M. (2015). Ética en las administraciones públicas: de los principios al marco institucional. Revista Vasca de Gestión de Personas y Organizaciones, 9, 8-17. https://apps.euskadi.eus/z16-a5app2/es/t59auUdaWar/ $\mathrm{R} 3 /$ verArticulo? numejem $=9 \&$ tipo $=\mathrm{R} \&$ seccion $=51 \&$ correlativo $=1 \&$ contenido $=1 \&$ locale $=e s$ 